\title{
COHOPFICITY OF SEIFERT-BUNDLE GROUPS
}

\author{
F. GONZÁLEZ-ACUÑA, R. LITHERLAND, AND W. WHITTEN
}

\begin{abstract}
A group $G$ is cohopfian, if every monomorphism $G \rightarrow G$ is an automorphism. In this paper, we answer the cohopficity question for the fundamental groups of compact Seifert fiber spaces (or Seifert bundles, in the current vernacular). If $M$ is a closed Seifert bundle, then the following are equivalent: (a) $\pi_{1} M$ is cohopfian; (b) $M$ does not cover itself nontrivially; (c) $M$ admits a geometric structure modeled on $S^{3}$ or on $\widetilde{\mathrm{SL}_{2} \mathbf{R}}$. If $M$ is a compact Seifert bundle with nonempty boundary, then $\pi_{1} M$ is not cohopfian.
\end{abstract}

An object $\mathbb{C}$ of a category is hopfian if any epimorphism from $\mathbb{C}$ to itself is an automorphism. Dually, $\mathbb{C}$ is cohopfian, if any monomorphism from $\mathbb{C}$ to itself is an automorphism. Thus, a group is cohopfian if and only if it cannot be properly imbedded in itself. A group is complete, if its automorphisms are all inner and its center is trivial. Finite groups and certain two-generator complete hopfian groups [MS] are cohopfian, while infinite f.g. abelian groups and free products of nontrivial groups are not cohopfian, for example.

In [GW], the following two results concerning the cohopficity of 3-manifold groups were obtained.

Theorem. Let $M$ be a Haken manifold, different from a collar, whose boundary is a nonempty union of incompressible tori. Then $\pi_{1} M$ is cohopfian if and only if the collection of those components of the characteristic submanifold meeting $\partial M$ is a disjoint union of collars.

Corollary. The group of a nontrivial knot $K$ is cohopfian if and only if $K$ is not a torus knot, a cable knot, or a composite knot.

Since, as we have remarked, free products and $Z$ are not cohopfian while finite groups are, the cohopficity question for the fundamental groups of closed 3-manifolds is reduced to the following.

Which closed irreducible 3-manifolds $M^{3}$ with infinite $\pi_{1}$ have a cohopfian fundamental group?

A sufficient (but not necessary) condition is that $M^{3}$ be either Haken with positive Gromov invariant or hyperbolic. Moreover, no subgroup $G$ of infinite index in $\pi_{1} M^{3}$ is isomorphic to $\pi_{1} M^{3}$ when $M^{3}$ is also $P^{2}$-irreducible, since $H_{3}\left(\pi_{1} M^{3} ; Z_{2}\right) \neq 0$ while $H_{3}\left(G ; Z_{2}\right)=0$ [GG].

Received by the editors April 24, 1991.

1991 Mathematics Subject Classification. Primary 20C99, 55R05, 55R10, 57M05, 57M10, $57 \mathrm{~N} 99$.

The National Science Foundation provided partial support to the second author.

NSF/LaSER Grant VSFP-04 provided partial support to the third author. 
In this paper, we settle the cohopficity question for the class of fundamental groups of compact Seifert fiber spaces. To do this, it is convenient to consider a compact 3-manifold as a Seifert "bundle" if and only if it admits a foliation by circles. We then divide the collection of closed 3-manifolds that can be foliated by circles into six classes with each manifold in a class admitting the same geometric structure. These classes do not overlap, and all the Seifert-bundle structures of a closed manifold admit the same geometric structure (cf. [Sc, $\S 4])$. This geometric subdivision allows us to prove our main result, Corollary 5.

Corollary 5. Let $M$ be a closed Seifert fiber space with base orbifold $B$. The following statements are equivalent.

(a) $\pi_{1} M$ is cohopfian.

(b) $M$ does not cover itself nontrivially.

(c) $M$ admits a geometric structure modeled on $S^{3}$ or on $\widetilde{\mathrm{SL}_{2} \mathbf{R}}$.

(d) $e(M) \cdot \bar{\chi}(B)=0$.

The paper concludes with the following two theorems.

Corollary 6. The fundamental group of a homology 3-sphere admitting a Seifert fibration is cohopfian.

Theorem 7. The fundamental group of a compact Seifert fiber space with nonempty boundary is not cohopfian.

We wish to thank R. J. Daverman for helpful conversations. The last author would also like to thank the Instituto de Matematicas of the Universidad Nacional Autonoma de Mexico along with Louisiana State University for their hospitality while part of this work was done.

\section{INTRODUCTION}

Following Scott [Sc, §3], we call a 3-manifold $M$ a Seifert bundle (or a Seifert fiber space), if $M$ admits a decomposition into disjoint circles (fibers) each having a regular neighborhood that is either a fibered solid torus or a fibered solid Klein bottle. With this definition, a compact 3-manifold admits a Seifert fibration if and only if it can be foliated by circles [Ep].

Now, let $M$ be a Seifert fiber space. An exceptional fiber of $M$ is either isolated, in which case it is the core of a fibered solid torus covered by a trivially fibered solid torus, or it belongs to a fibered solid Klein bottle $K$. The critical fibers of $K$ together form a 1-sided annulus, and so the collection of all exceptional fibers of $M$ is a set of isolated fibers along with 1-sided annuli, tori, and Klein bottles. It follows that, if $M$ is connected, the union of all regular fibers in $M$ forms a circle bundle over a surface.

We consider the base space $B$ of $M$ as a 2-dimensional orbifold that may contain cone points and reflector curves, but no corner reflectors. If $M$ is closed, then $B$ is an orbifold without boundary, where any boundary components of the underlying space $|B|$ of $B$ serve as reflector circles for $B$.

Associated with each isolated exceptional fiber of $M$ are the fiber's Seifert invariants, $(\alpha, \beta)$, where $\alpha$ and $\beta$ are relatively prime; we assume $\beta$ to be normalized so that $0<\beta<\alpha$, except where noted in the proof of Lemma 3. If 
$\left(\alpha_{1}, \beta_{1}\right), \ldots,\left(\alpha_{r}, \beta_{r}\right)$ are the Seifert invariants of $M$, then a natural invariant of the orbifold structure of $B$ is its orbifold Euler characteristic

$$
\bar{\chi}(B)=\chi(B)-\sum_{i=1}^{r}\left(1-\frac{1}{\alpha_{i}}\right) ;
$$

this equation is sometimes called the Riemann-Hurwitz formula. If $\widetilde{B} \rightarrow B$ is an orbifold covering of degree $m$, then $\bar{\chi}(\widetilde{B})=m \bar{\chi}(B)$.

The final invariant of the Seifert-bundle structure of $M$ that we shall need is the rational Euler number, $e(M)$. This is the obstruction to the existence of a "multifold section" in $M$-a surface $F$ in $M$ that is orthogonal to the (Seifert) fibers of $M$ and for which the restriction, $p \mid F: F \rightarrow B$, is an orbifold covering; here, $B$ is the base space of $M$, and $p: M \rightarrow B$ is the natural projection map inducing the orbifold structure of $B$. When $M$ is closed and orientable and has normalized Seifert invariants $\left(\alpha_{1}, \beta_{1}\right), \ldots,\left(\alpha_{r}, \beta_{r}\right)$, then

$$
e(M)=-b-\sum_{i=1}^{r} \frac{\beta_{i}}{\alpha_{i}}
$$

where $b$ is an integral invariant of the circle bundle obtained by removing the exceptional fibers of $M$. When $M$ is nonorientable, then $e(M)=0$ and $b \in Z_{2}$.

Now, if the base orbifold $B$ of $M$ is closed, there is a nice relationship between $e(M)$ and the rational Euler number, $e(\widetilde{M})$, of any finite covering, $\widetilde{M}$, of $M$. If $\widetilde{B}$ is the base orbifold of $\widetilde{M}$, then a covering $\Psi: \widetilde{M} \rightarrow M$ induces an orbifold covering $\psi: \widetilde{B} \rightarrow B$ such that the following diagram commutes:

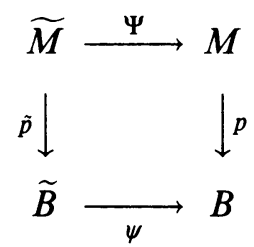

In this diagram, $\widetilde{p}$ and $p$ denote the natural projections. Suppose that the degree of $\psi: \widetilde{B} \rightarrow B$ is $m$ and the degree with which a regular fiber of $\widetilde{M}$ covers a regular fiber of $M$ is $n$. Then the degree of $\Psi: \widetilde{M} \rightarrow M$ is $m \cdot n$ and $e(\widetilde{M})=\frac{m}{n} e(M)$. See [NR, Theorem 1.2] for a sketch of the proof.

Scott has shown [Sc, Lemma 3.7] that if $M$ is compact, then $e(M)=0$ if and only if there exists a surface $F$ and a finite, fiber-preserving covering, $F \times S^{1} \rightarrow M$, whose group of covering transformations respects the product structure of $F \times S^{1}$. Thus, in the cases of interest to us with $M$ closed and $e(M)=0$, we see that $M$ has the structure of a bundle over a 1-dimensional orbifold that is either a circle with no singular points or a closed interval with two reflector points.

As discussed by Thurston [Th] and shown in detail by Scott [Sc, §4], each closed Seifert fiber space $M$ admits precisely one of six geometric structures. The geometric structure of $M$ does not change with different Seifert fibrations of the underlying space of $M$, and the geometry that $M$ admits depends only upon $e(M)$ and $\bar{\chi}(B)$. The six possibilities are shown in the following table from $[\mathrm{Sc}]$. 


\begin{tabular}{|c|c|c|c|}
\hline & $\bar{\chi}>0$ & $\bar{\chi}=0$ & $\bar{\chi}<0$ \\
\hline$e=0$ & $S^{2} \times \mathbf{R}$ & $E^{3}$ & $H^{2} \times \mathbf{R}$ \\
\hline$e \neq 0$ & $S^{3}$ & Nil & $\widetilde{\mathrm{SL}_{2} \mathbf{R}}$ \\
\hline
\end{tabular}

For details on the ideas touched upon in this introduction, we refer the reader to [Sc, S, BS, Th], and [O].

\section{COHOPFICITY RESULTS}

Theorem 1. Let $M$ be a closed Seifert fiber space with rational Euler number $e(M)=0$. Then $\pi_{1} M$ is not cohopfian. In fact, there exists a nontrivial covering $M \rightarrow M$.

Proof. It suffices to show that $M$ covers itself nontrivially. First notice that $M$ has a geometric structure modeled on exactly one of $S^{2} \times \mathbf{R}, E^{3}$, and $H^{2} \times \mathbf{R}$.

If $M$ is closed and admits a geometric structure modeled on $S^{2} \times \mathbf{R}$, then $M$ is homeomorphic to one of four possible manifolds, $P^{2} \times S^{1}, S^{2} \times S^{1}, P^{3} \# P^{3}$, and $S^{2} \tilde{\times} S^{1}$. Now, each of $P^{2} \times S^{1}, S^{2} \times S^{1}$, and $S^{2} \tilde{\times} S^{1}$ fibers over $S^{1}$, and $P^{3} \# P^{3}$ fibers over a closed interval with two reflector points. The three spaces that fiber over $S^{1}$ have periodic gluing maps (with period either 1 or 2), and so one easily constructs a nontrivial covering of each by itself.

Tollefson [To] has shown that $P^{3} \# P^{3}$ is an $n$-fold cover of itself for every positive integer $n$. To see, for that example, that $P^{3} \# P^{3}$ double-covers itself, think of $P^{3} \# P^{3}$ as $P^{3} \# S^{3} \# P^{3}$. There is then a two-fold covering, $\varphi: P^{3} \# S^{3} \# P^{3}$ $\rightarrow P^{3} \# P^{3}$, that takes each $P^{3}$-summand of $P^{3} \# S^{3} \# P^{3}$ homeomorphically onto the left $P^{3}$-summand of $P^{3} \# P^{3}$ and takes the $S^{3}$-summand onto the right $P^{3}$ summand of $P^{3} \# P^{3}$ as the usual double covering, $S^{2} \times I \rightarrow P^{2} \tilde{x} I$.

In the remaining case, when $M$ is geometrically modeled on either $E^{3}$ or $H^{2} \times \mathbf{R}$, we prefer an algebraic approach, which in fact can be adopted to the case when $M$ is modeled on $S^{2} \times \mathbf{R}$.

So, let $M$ be a closed Seifert bundle with base orbifold $B$ and rational Euler number $e(M)=0$. If the underlying space $|B|$ of $B$ of orientable, then $\pi_{1} M$ has a presentation with generators

$$
a_{1}, b_{1}, \ldots, a_{p}, b_{p}, q_{1}, \ldots, q_{n}, d_{1}, \ldots, d_{m}, y_{1}, \ldots, y_{m}, h
$$

and with relations

$$
\begin{gathered}
a_{i} h a_{i}^{-1}=h^{\varepsilon_{i}}, \quad b_{i} h b_{i}^{-1}=h^{\varepsilon_{i}^{\prime}}, \quad q_{i} h q_{i}^{-1}=h, \quad q_{i}^{\alpha_{i}} h^{\beta_{i}}=1, \\
d_{1} \cdots d_{m} \cdot q_{1} \cdots q_{n} \prod_{i=1}^{p}\left[a_{i}, b_{i}\right]=h^{b}, \quad y_{i}^{2}=h, \quad d_{i} y_{i} d_{i}^{-1}=y_{i}^{\varepsilon_{i}^{\prime \prime}} .
\end{gathered}
$$

If $|B|$ is nonorientable, then $\pi_{1} M$ has a presentation with generators

$$
v_{1}, \ldots, v_{k}, q_{1}, \ldots, q_{n}, d_{1}, \ldots, d_{m}, y_{1}, \ldots, y_{m}, h
$$

and with relations

$$
\begin{gathered}
v_{i} h v_{i}^{-1}=h^{\varepsilon_{i}}, \quad q_{i} h q_{i}^{-1}=h, \quad q_{i}^{\alpha_{i}} h^{\beta_{i}}=1, \\
d_{1} \cdots d_{m} \cdot q_{1} \cdots q_{n} \prod_{i=1}^{p} v_{i}^{2}=h^{b}, \quad y_{i}^{2}=h, \quad d_{i} y_{i} d_{i}^{-1}=y_{i}^{\varepsilon_{i}^{\prime \prime}}
\end{gathered}
$$


cf. [OVZ]. We shall refer to either of these presentations as presentation $\mathscr{P}$.

Here, the $y_{i}$ and $d_{i}$ occur only when $B$ has reflector circles. Also, when $|B|$ is orientable, each $\varepsilon_{i}$ and $\varepsilon_{i}^{\prime}$ is +1 when $M$ is orientable, and each is -1 , otherwise. When $|B|$ is nonorientable, each $\varepsilon_{i}$ is -1 when $M$ is orientable, and $\varepsilon_{1}$ is always +1 , otherwise.

Let $\alpha$ denote the least common multiple of the numbers $1, \alpha_{1}, \ldots, \alpha_{n}$, and let $r$ be an integer such that $r \equiv 1 \bmod (2 \alpha)(r \equiv-1 \bmod (2 \alpha)$ would also work) and $r>1$. Let $t_{i}$ denote the even integer $(1-r) \beta_{i} / \alpha_{i}$, and set $s=\frac{1}{2}\left((1-r) b+\sum_{i=1}^{n} t_{i}\right)$.

Now replace the generator $q_{i}$ by $q_{i} h^{t_{i}}$. In case $M$ is nonorientable, replace the generator $a_{1}$ by $a_{1} h^{s}$ (if $|B|$ is orientable and $p>0$ ) and the generator $v_{1}$ by $v_{1} h^{-s}$ (if $|B|$ is nonorientable). If $|B|$ is orientable and if $p=0$ and $m>0$, also replace the generator $d_{1}$ by $h^{-2 s} d_{1}$. Then we see that $\pi_{1} M$ has a presentation $\mathscr{P}^{\prime}$ with the same generators and relations as those of $\mathscr{P}$ above, except that $q_{i}^{\alpha_{i}} h^{\beta_{i}}=1$ is replaced by $q_{i}^{\alpha_{i}} h^{r \beta_{i}}=1$, and $h^{b}$ on the right hand side of the long relation is replaced by $h^{r b}$.

Next define a homomorphism $\varphi$ from $\|\mathscr{P}\|\left(=\pi_{1} M\right)$ to $\left\|\mathscr{P}^{\prime}\right\|\left(=\pi_{1} M\right)$ by sending $h$ to $h^{r}, y_{i}$ to $y_{i}^{r}$, and the remaining generators of $\mathscr{P}$ to the corresponding ones of $\mathscr{P}^{\prime}$. Recalling that $h$ is carried by a regular fiber of $M$ and generates an infinite, cycle, normal subgroup of $\pi_{1} M$ [Sc, Lemma 3.2], one sees that $\varphi$ is a monomorphism, but not an isomorphism, by using the diagram

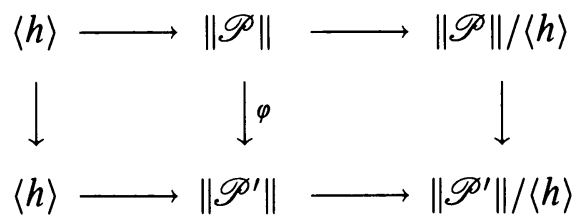

in which the vertical map on the far right is an isomorphism.

We now have an $r$-fold covering, $\widetilde{M} \rightarrow M$, induced by the monomorphism $\varphi: \pi_{1} M \rightarrow \pi_{1} M$, with $\pi_{1} \widetilde{M} \approx \pi_{1} M$. When $M$ is geometrically modeled on either $E^{3}$ or $H^{2} \times \mathbf{R}$, then $\widetilde{M}$ is modeled on the same geometry. Hence, in these cases, each of $M$ and $\widetilde{M}$ is $P^{2}$-irreducible (see [Sc, §3], say), and they are also sufficiently large, as is easy to see. It follows that $\widetilde{M} \cong M[\mathrm{Wa}, \mathrm{He}]$ (cf. [OVZ]).

Remarks. (1) Of the 10 closed, flat, 3-manifolds, there is only one that is the union of two twisted I-bundles; in that case, each of them is an orientable twisted I-bundle over the Klein bottle. As Daverman [Da] has shown, this union of $I$-bundles is an $n$-fold cover of itself for each odd $n$.

(2) Tollefson [To, p. 108] points out an infinite number of closed Seifert bundles that cover themselves nontrivially and, as it turns out, are all geometrically modeled on $H^{2} \times \mathbf{R}$.

Theorem 2. Let $M$ be a closed Seifert fiber space with $e(M) \neq 0$, and suppose that there exists a nontrivial covering, $\Psi: M \rightarrow M$. Then $M$ admits a geometric structure modeled on Nil.

Proof. Since $e(M) \neq 0$ and $\Psi: M \rightarrow M$ is nontrivial, $M$ is orientable, and the appropriate geometric structure for $M$ must be either Nil or $\widetilde{\mathrm{SL}_{2} \mathbf{R}}$. Hence, the universal covering space of $M$ is homeomorphic to $R^{3}$. Moreover, $M$ is 
not covered by $S^{1} \times S^{1} \times S^{1}$, since if it were, then $M$ would admit a geometric structure modeled on $E^{3}$ (see [Sc]), making $e(M)=0$. Therefore, $M$ admits a unique Seifert-bundle structure (up to isomorphism) [Sc, Theorem 3.8], and so we can assume that $\Psi: M \rightarrow M$ is fiber preserving.

Let $B$ denote the base orbifold of $M$. Since $M$ is closed, $B$ is a closed orbifold, and we have $e(M)=(m / n) e(M)$, where $m$ is the degree of the covering $\psi: B \rightarrow B$ induced by $\Psi$. Thus, $m=n$. Since $m \cdot n$ is the degree of $\Psi$, we have $m \cdot n>1$, and it follows that $m>1$. But since $\bar{\chi}(B)=m \bar{\chi}(B)$, we have $\bar{\chi}(B)=0$. Hence, $M$ is geometrically modeled on Nil.

Lemma 3. If $M$ is a closed Seifert fiber space admitting a geometric structure modeled on Nil, then $\pi_{1} M$ is not cohopfian.

Proof. Since $M$ is geometrically modeled on Nil, we have $\bar{\chi}(B)=0$, where $B$ is the base orbifold of $M$. Hence, $B$ is a torus or a Klein bottle with no cone points, $S^{2}$ with cone points of multiplicities $(2,2,2,2),(2,2,4)$, $(2,3,6)$, or $(3,3,3)$, or $B$ is $P^{2}$ with two cone points of multiplicities $(2,2)$ (see [Th] or [Sc]). Notice that $M$ is orientable, since $e(M) \neq 0$, and so $B$ can have no reflector circles, that is, the underlying space $|B|$ of $B$ must be closed. We prove the lemma case-by-case.

(1) $|B| \cong S^{1} \times S^{1}$.

$$
\pi_{1} M \approx\left\langle a_{1}, b_{1}, h: a_{1} h a_{1}^{-1}=h, b_{1} h b_{1}^{-1}=h,\left[a_{1}, b_{1}\right]=h^{b}\right\rangle,
$$

with $b \in Z$ and $b \neq 0$. The assignment, $a_{1} \mapsto a_{1}^{2}, b_{1} \mapsto b_{1}, h \mapsto h^{2}$, defines an endomorphism $\varphi: \pi_{1} M \rightarrow \pi_{1} M$, and we have a commutative diagram.

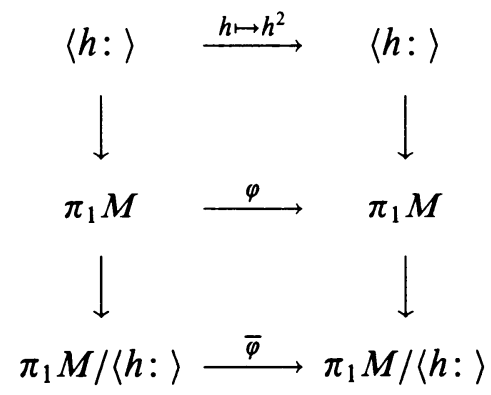

Since $\bar{\varphi}$, which is induced by $\varphi$, is clearly a monomorphism (as is easily checked) as is $h \mapsto h^{2}$, so is $\varphi$. Also, $\varphi$ is not an epimorphism.

(2) $|B| \cong$ Klein bottle.

$$
\pi_{1} M \approx\left\langle v_{1}, v_{2}, h: v_{1} h v_{1}^{-1}=h^{-1}, v_{2} h v_{2}^{-1}=h^{-1}, v_{1}^{2} v_{2}^{2}=h^{b}\right\rangle,
$$

with $b \in Z$ and $b \neq 0$. The assignment, $v_{1} \mapsto v_{1}^{3}, v_{2} \mapsto v_{2}^{3}, h \mapsto h^{3}$, defines an endomorphism $\varphi: \pi_{1} M \rightarrow \pi_{1} M$, and one can show by the same method as in case (1) that $\varphi$ is a monomorphism but not an epimorphism.

(3) $|B| \cong S^{2}$ or $P^{2}$.

We shall use the following notation throughout the remainder of the proof. We let $B_{0}$ denote $|B|$ punctured at the cone points. The number of exceptional fibers of $M$ is $k(=2,3$, or 4$)$, and $\alpha_{i}$ and $\beta_{i}$ have their usual meaning except that we take $b=0$ and, thus, do not assume that $0<\beta_{i}<\alpha_{i}$.

The notation of various groups of interest is as follows.

$$
\pi_{1}\left(B_{0}\right)= \begin{cases}\left\langle q_{1}, \ldots, q_{k}: q_{1} \cdots q_{k}=1\right\rangle & \text { if }|B| \cong S^{2}, \\ \left\langle v, q_{1}, q_{2}: v^{2} q_{1} q_{2}=1\right\rangle & \text { if }|B| \cong P^{2} .\end{cases}
$$


As in cases (1) and (2), we let $h$ denote the element of $\pi_{1} M$ represented by a regular fiber so that

$$
\pi_{1} M=\pi_{1} B_{0} \ltimes\langle h:\rangle /\left\langle q_{i}^{\alpha_{i}} h^{\beta_{i}}(i=1, \ldots, h)\right\rangle ;
$$

the action of $\pi_{1} B_{0}$ on $\langle h:\rangle$ is $q_{i} h q_{i}^{-1}=h \quad(i=1, \ldots, k)$ and $v h v^{-1}=h^{-1}$ (if present). The orbifold fundamental group of $B$ is

$$
\bar{\pi}_{1} B=\pi_{1} M /\langle h:\rangle=\pi_{1} B_{0} /\left\langle q_{i}^{\alpha_{i}}(i=1, \ldots, k)\right\rangle .
$$

An orbifold covering $\tau: B \rightarrow B$ of degree $n$ will be called good, if each cone point is covered by one cone point of the same multiplicity and some regular points. (Note that there are bad coverings: in the case $(3,3,3)$, for instance, take a cyclic 3 -fold covering of $S^{2}$ branched over two of its cone points.) A good covering corresponds to a transitive representation $\rho: \pi_{1} B_{0} \rightarrow \Sigma_{n}$ such that each $\rho\left(q_{i}\right)$ has one fixed point and some number $m_{i}$ of cycles of length $\alpha_{i}$; hence, $n=m_{i} \alpha_{i}+1$. We show first that good coverings exist in each case.

$(2,2,2,2) \quad$ For any $n \equiv 1(2)$,

$$
\begin{gathered}
q_{1}, q_{2} \mapsto(12)(34) \cdots(n-2, n-1), \\
q_{3}, q_{4} \mapsto(23)(45) \cdots(n-1, n) .
\end{gathered}
$$

$(2,4,4) \quad$ For $n=5$,

$$
q_{1} \mapsto(12)(34), \quad q_{2} \mapsto(1345), \quad \text { and } \quad q_{3} \mapsto(1532)
$$

$(2,3,6) \quad$ For $n=7$,

$$
q_{1} \mapsto(12)(34)(56), \quad q_{2} \mapsto(136)(275), \quad \text { and } \quad q_{3} \mapsto(132657) .
$$

$(3,3,3) \quad$ For $n=4$,

$$
\begin{aligned}
& q_{1} \mapsto(123), \quad q_{2} \mapsto(134), \quad \text { and } \quad q_{3} \mapsto(142) . \\
& \text { For } n=7, \\
& q_{1} \mapsto(123)(456), \quad q_{2} \mapsto(247)(365), \quad \text { and } \quad q_{3} \mapsto(134)(276) .
\end{aligned}
$$

$(2,2) \quad$ For any $n \equiv 1(2)$,

$$
\begin{gathered}
v \mapsto(n(n-1) \cdots 1), \\
q_{1} \mapsto(1(n-1))(3(n-3)) \cdots((n-2) 2),
\end{gathered}
$$

and

$$
q_{2} \mapsto(3(n-1))(5(n-3)) \cdots(n 2) .
$$

We first consider the case $|B|=S^{2}$. Let $\tau: B \rightarrow B$ be a good covering. Then $\tau$ restricts to a covering $\widetilde{B}_{0} \rightarrow B_{0}$.

We can choose generators $q_{i p}\left(i=1, \ldots, k ; p=0, \ldots,(n-1) / \alpha_{i}\left(=m_{i}\right)\right)$ for $\widetilde{B}_{0}$ so that

$$
\pi_{1} \widetilde{B}_{0}=\left\langle q_{i p}: \prod_{i=1}^{k} \prod_{p=0}^{m_{i}} q_{i p}=1\right\rangle
$$

and $\tau_{*}\left(q_{i 0}\right)=g_{i} q_{i} g_{i}^{-1}, \tau_{*}\left(q_{i p}\right)=g_{i p} q_{i}^{\alpha_{i}} g_{i p}^{-1}$, for $p \geq 1$. 
Note that since the relation

$$
\prod_{i=1}^{k}\left[g_{i} q_{i} g_{i}^{-1} \prod_{p=1}^{m_{i}} g_{i p} q_{i}^{\alpha_{i}} g_{i p}^{-1}\right]=1
$$

holds in $\pi_{1} B_{0}$, it certainly holds in $\pi_{1} M$. But in $\pi_{1} M$, we also have $q_{i}^{\alpha_{i}}=$ $h^{-\beta_{i}}(i=1, \ldots, k)$, and so

$$
\prod_{i=1}^{k} g_{i} q_{i} g_{i}^{-1}=h^{\sum_{i=1}^{k} m_{i} \beta_{i}}
$$

in $\pi_{1} M$.

Now define $\varphi: \pi_{1} M \rightarrow \pi_{1} M$ by

$$
\varphi\left(q_{i}\right)=g_{i} q_{i} g_{i}^{-1} h^{-m_{i} \beta_{i}}, \quad \varphi(h)=h^{n} .
$$

We must check that this is well defined.

Certainly $\left[\varphi\left(q_{i}\right), \varphi(h)\right]=1$. Also,

$$
\begin{aligned}
\left(\varphi\left(q_{i}\right)\right)^{\alpha_{i}} \cdot(\varphi(h))^{\beta_{i}} & =g_{i} q_{i}^{\alpha_{i}} g_{i}^{-1} h^{-m_{i} \alpha_{i} \beta_{i}} h^{n \beta_{i}} \\
& =g_{i} q_{i}^{\alpha_{i}} g_{i}^{-1} h^{\beta_{i}} \quad\left(\text { since } n=m_{i} \alpha_{i}+1\right) \\
& =1 .
\end{aligned}
$$

Finally,

$$
\varphi\left(q_{1}\right) \cdots \varphi\left(q_{k}\right)=\left(\prod_{i=1}^{k} g_{i} q_{i} g_{i}^{-1}\right) h^{-\sum_{i=1}^{k} m_{i} \beta_{i}}=1
$$

by relation $(*)$.

We now have a commutative diagram:

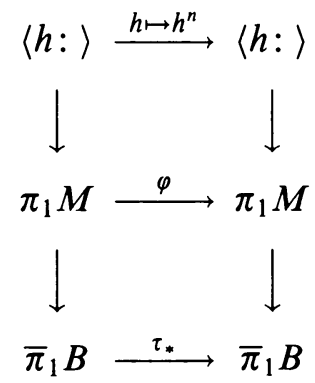

Since $h \mapsto h^{n}$ and $\tau_{*}$ are monomorphisms, so is $\varphi$. It is also clear that $\varphi$ is not an epimorphism.

Suppose now that $|B|=P^{2}$. Let $\tau: B \rightarrow B$ be the good orbifold covering of degree 3 corresponding to the transitive representation $\rho: \pi_{1} B_{0} \rightarrow \Sigma_{3}$ given by $\rho(v)=\left(\begin{array}{lll}3 & 2 & 1\end{array}\right), \rho\left(q_{1}\right)=\left(\begin{array}{ll}1 & 2\end{array}\right), \rho\left(q_{2}\right)=\left(\begin{array}{ll}2 & 3\end{array}\right)$. Much as before, we have

$$
\pi_{1} \widetilde{B}_{0}=\left\langle\tilde{v}, q_{10}, q_{11}, q_{20}, q_{21}: \tilde{v}^{2} q_{11} q_{21} q_{10} q_{20}=1\right\rangle
$$

and $\tau_{*}(\tilde{v})=v^{3}, \tau_{*}\left(q_{10}\right)=q_{2}^{-1} q_{1} q_{2}, \tau_{*}\left(q_{20}\right)=\left(q_{1} q_{2}\right)^{-2} q_{2}\left(q_{1} q_{2}\right)^{2}, \tau_{*}\left(q_{11}\right)=q_{1}^{2}$, $\tau_{*}\left(q_{21}\right)=q_{2}^{2}$.

Now define $\varphi: \pi_{1} M \rightarrow \pi_{1} M$ by

$$
\begin{aligned}
\varphi(v) & =v^{3}, \quad \varphi\left(q_{1}\right)=q_{2}^{-1} q_{1} q_{2} h^{-\beta_{1}} \\
\varphi\left(q_{2}\right) & =\left(q_{1} q_{2}\right)^{-2} q_{2}\left(q_{1} q_{2}\right)^{2} h^{-\beta_{2}}, \quad \text { and } \varphi(h)=h^{3}
\end{aligned}
$$


The proof that $\varphi$ is well defined and is a monomorphism, but not an epimorphism, is exactly as with $|B|=S^{2}$; note, however, that it is important that conjugating elements are orientation preserving and thus commute with $h$. We check directly that $(\varphi(v))^{2} \cdot \varphi\left(q_{1}\right) \cdot \varphi\left(q_{2}\right)=1$.

$$
\begin{aligned}
(\varphi(v))^{2} \cdot \varphi\left(q_{1}\right) \cdot \varphi\left(q_{2}\right) & =v^{6} q_{2}^{-1} q_{1} q_{2}\left(q_{1} q_{2}\right)^{-2} q_{2}\left(q_{1} q_{2}\right)^{2} h^{-\beta_{1}-\beta_{2}} \\
& =v^{6} q_{2}^{-2} h^{-\beta_{2}} q_{1}^{-1} h^{-\beta_{1}} q_{2}\left(q_{1} q_{2}\right)^{2} \\
& =v^{6} q_{1} q_{2}\left(q_{1} q_{2}\right)^{2}=1 .
\end{aligned}
$$

Remark. For $M$ with exceptional fibers as in Lemma 3, one can obtain specific imbeddings, $\pi_{1} M \rightarrow \pi_{1} M$, by drawing a covering $\widetilde{B}_{0}$ of $B_{0}$ and using it to obtain the $g_{i}$ 's.

Recall that a closed Seifert fiber space $M$ is small, if it is geometrically modeled on either $S^{2} \times R$ or $S^{3}$, or if it is a circle bundle over a torus or a Klein bottle [O, p. 91]. If $M$ is not small, then it is large.

Proposition 4. Let $M$ be a closed Seifert fiber space. Then $\pi_{1} M$ is not cohopfian if and only if there exists a nontrivial covering $M \rightarrow M$.

Proof. The condition is obviously sufficient, so suppose that $\pi_{1} M$ is not cohopfian. If $e(M)=0$, then $M$ covers itself nontrivially (Theorem 1).

If $e(M) \neq 0$, then $M$ must be geometrically modeled on either Nil or $\widetilde{\mathrm{SL}_{2} \mathbf{R}}$; it cannot be modeled on $S^{3}$, since otherwise $\pi_{1} M$ would be finite and, therefore, cohopfian. If $M$ admits a geometric structure modeled on Nil, then the base orbifold of $M$ is one of the following seven.

(1) $|B| \cong S^{1} \times S^{1}$ and $B$ has no cone points;

(2) $|B| \cong$ Klein bottle and $B$ has no cone points;

(3) $|B| \cong S^{2}$ and $B$ has 4 cone points with multiplicities $(2,2,2,2)$;

(4) $|B| \cong P^{2}$ and $B$ has 2 cone points with multiplicities $(2,2)$;

(5) $|B| \cong S^{2}$ and $B$ has 3 cone points with multiplicities $(2,4,4),(2,3,6)$, or $(3,3,3)$.

Now $M$ is orientable (since $e(M) \neq 0$ ), and thus if the base orbifold of $M$ is one of the first four, (1), (2), (3), (4) above, then $M$ must be a Haken manifold (see [J, Theorem VI.15, p. 96], for example). If the base orbifold of $M$ is listed under (3), (4), or (5), then $M$ is large. Thus, $\pi_{1} M$ determines $M$ up to homeomorphism among closed, orientable, irreducible 3-manifolds when $M$ is Haken [Wa], or among large Seifert bundles when $M$ is large ([OVZ]; cf. $[\mathrm{O}$, pp. 97, 134]).

Let $\widetilde{M}$ denote the covering space of $M$ corresponding to a nontrivial imbedding of $\pi_{1} M$ in itself. Since the universal covering space of $M$ is $R^{3}$ (and, thus, not $S^{2} \times R$ ), $M$ is $P^{2}$-irreducible (see [Sc, §3], say), and so $\pi_{1} M$ cannot be imbedded in itself with infinite index, as we pointed out in the opening remarks of the paper. Hence, the covering $\widetilde{M} \rightarrow M$ is finite, and $\widetilde{M}$ is a closed, orientable, Seifert bundle geometrically modeled on Nil (and, therefore, irreducible). Thus, $\widetilde{M}$ is either large or Haken, and it follows from remarks in the last paragraph that $\widetilde{M} \cong M$, since $\pi_{1} \widetilde{M} \approx \pi_{1} M$.

Suppose now that $M$ admits a geometric structure modeled on $\widetilde{\mathrm{SL}_{2} \mathbf{R}}$ and that $\pi_{1} M$ is not cohopfian. Let $G<\pi_{1} M$ be a proper subgroup isomorphic to $\pi_{1} M$. As in the case when $M$ was geometrically modeled on Nil, we see that 
$M$ is $P^{2}$-irreducible, and so $\left[\pi_{1} M: G\right]<\infty$. Let $\widetilde{M}$ denote the covering space of $M$ corresponding to $G$. Then $\widetilde{M}$ is a closed, orientable, Seifert bundle modeled on $\widetilde{\mathrm{SL}_{2} \mathbf{R}}$. Clearly, both $M$ and $\widetilde{M}$ are large, and so $\widetilde{M} \cong M$, since $\pi_{1} \widetilde{M} \approx \pi_{1} M$. But no Seifert fiber space admitting a geometric structure modeled on $\mathrm{SL}_{2} \mathbf{R}$ can cover itself nontrivially (Theorem 2). Therefore, $\pi_{1} M$ is cohopfian.

Remark. In the above proof, we do not need the powerful result of Scott [ $\left[\mathrm{Sc}_{1}\right]$ : If $M$ and $N$ are closed, orientable, irreducible 3-manifolds with infinite fundamental groups, if $N$ is Seifert fibered, and if $\pi_{1} M \approx \pi_{1} N$, then $M \cong N$.

Corollary 5. Let $M$ be a closed Seifert fiber space with base orbifold $B$. The following statements are equivalent.

(a) $\pi_{1} M$ is cohopfian.

(b) $M$ does not cover itself nontrivially.

(c) $M$ admits a geometric structure modeled on $S^{3}$ or on $\widetilde{\mathrm{SL}_{2} \mathbf{R}}$.

(d) $e(M) \cdot \bar{\chi}(B) \neq 0$.

Proof. Statements (a) and (b) are equivalent, by Proposition 4. That (c) $\Rightarrow$ (b) follows from Theorem 2. If (a) holds, then $e(M) \neq 0$, by Theorem 1, but if $M$ were geometrically modeled in Nil, then $\pi_{1} M$ would not be cohopfian, by Lemma 3. Therefore, (a) $\Rightarrow$ (c). Finally, the Scott-Thurston chart given at the end of $\S 1$ implies that (c) and (d) are equivalent.

Recall from [S, Theorem 12] that if $M$ is a closed Seifert fiber space that is also a homology 3-sphere different from $S^{3}$, then the base orbifold of $M$ consists of $S^{2}$ with $r$ cone points of multiplicities $\left(\alpha_{1}, \alpha_{2}, \ldots, \alpha_{r}\right)$, where $r \geq$ 3 and the multiplicities are pairwise relatively prime. Moreover, there is only one such homology sphere with finite fundamental group; its cone multiplicities are $(2,3,5)$ and the space itself is geometrically modeled on $S^{3}$, of course.

Corollary 6. The fundamental group of a homology 3-sphere admitting a Seifert fibration is cohopfian.

Proof. Let $M$ denote such a 3-manifold different from $S^{3}$, with base orbifold $B$. A presentation for $\pi_{1} M$ is

$$
\begin{aligned}
& \left\langle q_{1}, \ldots, q_{r}, h: q_{1} h q_{1}^{-1}=h, \ldots, q_{r} h q_{r}^{-1}=h,\right. \\
& \left.\quad q_{1}^{\alpha_{1}} h^{\beta_{1}}=1, \ldots, q_{r}^{\alpha_{r}} h^{\beta_{r}}=1, q_{1} \cdots q_{r}=h^{b}\right\rangle,
\end{aligned}
$$

where $r \geq 3$. The corresponding relation matrix for $H_{1}(M)$ is square and its determinant is

$$
D=b \alpha_{1} \cdots \alpha_{r}+\beta_{1} \alpha_{2} \cdots \alpha_{r}+\cdots+\alpha_{1} \alpha_{2} \cdots \alpha_{r-1} \beta_{r} ;
$$

therefore,

$$
\frac{D}{\alpha_{1} \cdots \alpha_{r}}=-e(M)
$$

Since $M$ is a homology sphere, we have $D= \pm 1$, and so $e(M) \neq 0$.

On the other hand,

$$
\bar{\chi}(B)=2-\sum_{i=1}^{r}\left(1-\frac{1}{\alpha_{i}}\right)=(2-r)+\sum_{i=1}^{r} \frac{1}{\alpha_{i}},
$$


and since $\alpha_{1}, \ldots, \alpha_{r}$ are pairwise relatively prime, $\sum 1 / \alpha_{i}$ is not an integer. Hence, $\bar{\chi}(B) \neq 0$, and so $M$ is geometrically modeled on either $S^{3}$ or $\widetilde{\mathrm{SL}_{2} \mathbf{R}}$. Since $\pi_{1} S^{3}$ is cohopfian, the result now follows from Corollary 5 .

Our final result settles the cohopficity question for $\pi_{1} M$ when $M$ is Seifert fibered and compact, and $\partial M \neq \varnothing$.

Theorem 7. The fundamental group of a compact Seifert fiber space with nonempty boundary is not cohopfian.

Proof. Let $M$ denote a compact Seifert fiber space with nonempty boundary. Then each component of $\partial M$ is either a torus or a Klein bottle. We can assume that $M$ is neither a solid torus nor a solid Klein bottle, and so each component of $\partial M$ is incompressible (see [Sc, Corollary 3.3], say). We can also assume that $M$ is not an $I$-bundle (trivial or not) over a torus or over a Klein bottle, since then $\pi_{1} M$ is isomorphic to either $Z \times Z$ or $\left\langle v, h: v h v^{-1}=h^{-1}\right\rangle$; the latter imbeds in itself (with index 2) by $v \mapsto v, h \mapsto h^{2}$.

Before attacking the general case, it is convenient to consider three more special ones. For these, $M$ is obtained from a solid Klein bottle $K$ by removing some (open) fibered solid Klein bottles and perhaps one (open) fibered solid torus from $\operatorname{Int}(K)$, and possibly replacing one regular fiber of $K$ by an exceptional one. We describe the cases in terms of the base orbifold $B$ of $M$.

Case 1. $|B|=D^{2} ; \partial(|B|)$ contains at least two reflector lines; $B$ has no cone points.

Case 2. $|B|=S^{1} \times I$; one component of $\partial(|B|)$ contains no singular points while the other contains at least one reflector line; $B$ has no cone points.

Case 3. $|B|=D^{2} ; \partial(|B|)$ contains at least one reflector line; $B$ has exactly one cone point.

Let $r$ denote the number of reflector lines in each case. Then, in Cases 1,2, or $3, \pi_{1} M$ is isomorphic to

$$
\begin{gathered}
\left\langle x_{1}, \ldots, x_{r}: x_{1}^{2}=x_{2}^{2}=\cdots=x_{r}^{2}\right\rangle, \\
\left\langle x_{1}, \ldots, x_{r}, t_{1}: x_{1}^{2}=\cdots=x_{r}^{2}, t_{1} x_{r}^{2} t_{1}^{-1}=x_{r}^{2}\right\rangle,
\end{gathered}
$$

or

$$
\left\langle x_{1}, \ldots, x_{r}, q_{1}: x_{1}^{2}=\cdots=x_{r}^{2}, q_{1} x_{r}^{2} q_{1}^{-1}=x_{r}^{2}, q_{1}^{\alpha} x_{r}^{2 \beta}=1\right\rangle,
$$

respectively. In each case, each $x_{i}^{2}$ is represented by a regular fiber of $M$ and generates an infinite, cyclic, normal subgroup of $\pi_{1} M$. In Case 1, a proper imbedding is induced by $x_{i} \mapsto x_{i}^{3}$. In Cases 2 and 3, we let $c_{1}, c_{2}, \ldots, c_{r+1}$ denote $x_{1}, x_{2}, \ldots, x_{r}, t_{1}$ or $x_{1}, \ldots, x_{r}, q_{1}$, respectively, and send $x_{1} \mapsto$ $\left(c_{2} c_{1}\right)^{-2} x_{1}\left(c_{2} c_{1}\right)^{2}, x_{i} \mapsto x_{i} \quad(i>1)$, and $t_{1} \mapsto t_{1}$ (Case 2) or $q_{1} \mapsto q_{1}$ (Case 3 ); these are similar to the endomorphism used in the proof of Lemma 2.4 of [GW]; notice that $\left(c_{2} c_{1}\right)^{2}$ commutes with each $x_{i}^{2}$. Now,

$$
\pi_{1} M /\left\langle x_{1}^{2}:\right\rangle \approx Z_{2} * \cdots * Z_{2} * Z, \quad \text { in Case } 2,
$$

and

$$
\pi_{1} M /\left\langle x_{1}^{2}:\right\rangle \approx Z_{2} * \cdots * Z_{2} * Z_{\alpha}, \quad \text { in Case 3, }
$$

with $\alpha \geq 2$ and with $r$ copies of $Z_{2}$ in each case. We then have a commutative diagram

$$
\begin{array}{ccccc}
\left\langle x_{1}^{2}\right\rangle & \rightarrow & \pi_{1} M & \rightarrow & \pi_{1} M /\left\langle x_{1}^{2}\right\rangle \\
\text { id } \downarrow & & \varphi \downarrow & & \downarrow \varphi \\
\left\langle x_{1}^{2}\right\rangle & \rightarrow & \pi_{1} M & \rightarrow & \pi_{1} M /\left\langle x_{1}^{2}\right\rangle
\end{array}
$$


where $\hat{\varphi}$ is induced by $\varphi$. Since $\hat{\varphi}$ is a monomorphism, but not an automorphism, so is $\varphi$.

In the general case, we let $A_{1}, \ldots, A_{d}$ denote (closed) annular neighborhoods (without cone points) of those boundary components of $|B|$ that are either reflector circles or contain reflector lines of $B$. Set $\left|B_{0}\right|=$ $\operatorname{cl}\left(|B|-\left(A_{1} \cup \cdots \cup A_{d}\right)\right)$, let $M_{0}$ denote the Seifert bundle in $M$ over $B_{0}$, and let $N_{i}$ denote the Seifert bundle over $A_{i}(i=1, \ldots, d)$. There are two cases.

First, suppose that some boundary component of $|B|$ and of $A_{1}$, say, contains reflector lines of $B$, and let $M_{1}=\operatorname{cl}\left(M-N_{1}\right)$. Then

$$
\pi_{1} M \approx \pi_{1} M_{1} *_{Z \times Z} \pi_{1} N_{1}
$$

where we are assuming that $M$ is not one of the special cases, 1, 2, or 3, above, so that the inclusion map $M_{1} \cap N_{1} \rightarrow M_{1}$ induces a proper imbedding, $Z \times Z \approx \pi_{1}\left(M_{1} \cap N_{1}\right) \rightarrow \pi_{1} M_{1}$. Notice that the amalgamating subgroup $Z \times Z$ also properly injects into $\pi_{1} N_{1}$. Now $\pi_{1} N_{1}$ is isomorphic to

$$
\left\langle x_{1}, \ldots, x_{r}, t_{1}: x_{1}^{2}=\cdots=x_{r}^{2}, t_{1} x_{r}^{2} t_{1}^{-1}=x_{r}^{2}\right\rangle,
$$

as in special Case 2, and the proper imbedding of this group that we gave clearly extends to a proper imbedding $\pi_{1} M \rightarrow \pi_{1} M$ whose restriction to $\pi_{1} M_{1}$ is the identity. (Here, we take the base point of $\pi_{1} M$ in $M_{1} \cap N_{1}$.)

Assume now that no boundary component of $|B|$ contains reflector lines. Then, one component of each $A_{i}$ is a reflector circle, and $\partial(|B|)$ contains at least one component that is not a reflector circle. Moreover, each fiber of the Seifert bundle $M_{0}$ over $B_{0}$ has a fibered solid torus as a regular neighborhood, and a component of $\partial M$ is either an incompressible torus or Klein bottle. We now show that $\pi_{1} M_{0}$ is not cohopfian.

Let $T_{1}, \ldots, T_{m}$ be the boundary components of $M_{0}$, and let $T_{m}$ be a boundary component not projecting to a boundary component of any $A_{i}$. Let $n$ be the number of exceptional fibers of $M_{0}$ (all isolated now), and let $r$ be the rank of $\pi_{1}\left(\left|B_{0}\right|\right)$. Then $\pi_{1} M$ has a presentation with generators $t_{j}$ $(1 \leq j<m), q_{i}(1 \leq i \leq n), a_{k} \quad(1 \leq k \leq r-m+1)$, and $h$ and with relations $t_{j} h t_{j}^{-1}=h^{\varepsilon_{j}^{\prime}},\left[q_{i}, h\right]=1, a_{k} h a_{k}^{-1}=h^{\varepsilon_{k}}$, and $q_{i}^{\alpha_{i}} h^{\beta_{i}}=1$, where $\varepsilon_{j}^{\prime}$ and $\varepsilon_{k}$ belong to $\{-1,1\}$. Notice that these generators and relations take into account all the possible combinations of orientability states for $M_{0},\left|B_{0}\right|$, and the various boundary components of $M_{0}$ (see [OVZ, p. 51]).

Let $\left\{c_{1}, \ldots, c_{r+n}\right\}$ be the set of generators $\left\{t_{1}, \ldots, t_{m-1}, q_{1}, \ldots, q_{n}, a_{1}\right.$, $\left.\ldots, a_{r-m+1}\right\}$; we can assume that $r+m \geq 2$. Let $\omega=\left(c_{2} c_{1}\right)^{2}$, and notice that $[\omega, h]=1$. Define $\varphi: \pi_{1} M_{0} \rightarrow \pi_{1} M_{0}$ by $\varphi\left(c_{1}\right)=\omega^{-1} c_{1} \omega, \varphi\left(c_{i}\right)=c_{i}(i>1)$, and $\varphi(h)=h$. Then

$$
\pi_{1} M_{0} /\langle h:\rangle \approx F_{r} * Z_{\alpha_{1}} * \cdots * Z_{\alpha_{n}}
$$

and the proof that $\varphi$ is a monomorphism but not an automorphism is similar to that for special Cases 2 and 3 and for the endomorphism $\varphi$ in the proof of Lemma 2.4 of [GW].

Finally, if a nonorientable, twisted $I$-bundle, $N_{1}$ say, meets $\partial M_{0}$ along the surface $T_{1}$ associated with the loop $t_{1} \quad\left(=c_{1}\right)$ we take the basepoint $*$ of $\pi_{1} M$ in $T_{1}$ and set $\varphi(x)=\omega^{-1} x \omega$ for each generator $x$ of $\pi_{1} N_{1}<\pi_{1} M$, and we define $\varphi$ on every other $\pi_{1} N_{i}$ to be the identity; an element of $\pi_{1} N_{i}$ would 
here be represented by a path from $*$ to a point $p_{i}$ on $\partial N_{i}$, then a loop in $N_{i}$, and back to $*$ along the original path from $*$ to $p_{i}$. We define $\varphi$ on $\pi_{1} M_{0}$ as in the preceding paragraph. Thus, we have a monomorphism $\varphi$ of $\pi_{1} M$ that is not an automorphism.

\section{REFERENCES}

[BS] F. Bonahon and L. Siebenmann, The classification of Seifert fibered 3-orbifolds, LMS Lecture Note Ser., 95, London Math. Soc., 1985, pp. 19-85.

[Da] R. J. Daverman, Manifolds with finite first homology as codimension 2 fibrators, preprint.

[Ep] D. B. A. Epstein, Periodic flows on 3-manifolds, Ann. of Math. (2) 95 (1972), 66-82.

[GG] J. C. Gómez-Larrañaga and F. González-Acuña, Lusternik-Schnirelmann category of 3manifolds, Topology (to appear).

[GW] F. González-Acuña and W. Whitten, Imbeddings of three-manifold groups, Mem. Amer. Math. Soc. No. 474, 1992.

[He] W. Heil, On P2-irreducible 3-manifolds, Bull. Amer. Math. Soc. 75 (1969), 772-775.

[J] W. Jaco, Lectures on three-manifold topology, CBMS Regional Conf. Ser. in Math., vol. 43, Amer. Math. Soc., Providence, R.I., 1980.

[MS] C. F. Miller and P. E. Schupp, Embeddings into Hopfian groups, J. Algebra 17 (1971), 171-176.

[NR] W. D. Neumann and F. Raymond, Seifert manifolds, plumbing, $\mu$-invariants and orientation reversing maps, Lecture Notes in Math., vol. 664, Springer-Verlag, Berlin and New York, pp. 162-195.

[O] P. Orlik, Seifert manifolds, Lecture Notes in Math., vol. 291, Springer-Verlag, Berlin and New York, 1972.

[OVZ] P. Orlik, E. Vogt, and H. Zieschang, Zur Topolgie gefasterter dreidimensionaler Mannigfaltigkeiten, Topology 6 (1967), 49-64.

[Sc] P. Scott, The geometries of 3-manifolds, Bull. London Math. Soc. 15 (1983), 401-487.

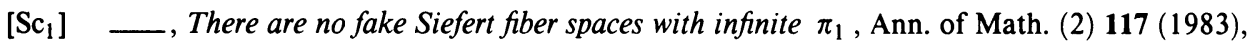
35-70.

[S] H. Seifert, Topologie dreidimensionaler gefaserter Räume, Acta Math. 60 (1933), 147-238.

[Th] W. P. Thurston, The geometry and topology of 3-manifolds, Lecture Notes, Princeton Univ., 1977.

[To] J. Tollefson, On 3-manifolds that cover themselves, Michigan Math. J. 16 (1969), 103-109.

[Wa] F. Waldhausen, On irreducible 3-manifolds which are sufficiently large, Ann. of Math. (2) 87 (1968), 56-88.

Instituto de Matematicas Universidad Nacional Autonoma de Mexico, Mexico, D.F. 04510

Department of Mathematics, Louisiana State University, Baton Rouge, Louisiana 70803

Department of Mathematics, University of Southwestern Louisiana, Lafayette, LOUISIANA 70504

E-mail address: whitten@usl.edu 